\title{
Mecanismos de articulación de las universidades con el estado-regulador, los mercados y la sociedad civil para asegurar la calidad de la educación superior
}

\author{
Milena Soto-Montoya ${ }^{1,2}$ \\ (1) Universidad de Baja California, Nayarit, México \\ (2) Fundación Universitaria María Cano, Medellín, Colombia (correo-e: imilenasotom@gmail.com)
}

Recibido May. 3, 2021; Aceptado Jun. 29, 2021; Versión final Ago. 16, 2021, Publicado Dic. 2021

\begin{abstract}
Resumen
Se propone una nueva taxonomía de instrumentos y mecanismos de articulación de las universidades con el Estado-Regulador, los mercados y la sociedad civil; que permitirían el incremento de la calidad tanto de las universidades como de los sistemas de educación superior. La metodología consiste en una actualización del Triángulo de Clark. Se discute que en un país donde un porcentaje bajo de las Instituciones de Educación Superior (IES) públicas estén acreditadas, pone en duda la convicción del propio Estado-Regulador en el valor de la acreditación institucional para asegurar la calidad de las IES. El modelo propuesto permite entender mejor cómo las IES, y de modo especial las universidades, pueden construir, cuidar y gestionar la reputación institucional. Se concluye que la universidad que sea activa en comunicar la calidad alcanzada, también influirá en la construcción de la reputación institucional.
\end{abstract}

Palabras clave: educación superior; gestión de la calidad; control social; gobernanza universitaria; modelo Clark-Engwall-Soto

\section{Contribution of national accreditation systems to ensuring the quality of higher education institutions: a conceptual theoretical approach}

\begin{abstract}
A new taxonomy of instruments and mechanisms for articulation of universities with the State-Regulator, markets and civil society which would allow the quality of both universities and higher education systems to be increased, is proposed. The methodology consists of an update of the Clark Triangle. It is also observed that in a country where a low percentage of public universities are accredited, it calls into question the conviction of the State-Regulator itself in the value of institutional accreditation to ensure the quality of them. The proposed model allows us to better understand how higher education institutions, and especially universities, can build caring and manage institutional reputation. It is found that universities that are active in communicating the quality achieved, will also influence the construction of institutional reputation.
\end{abstract}

Keywords: higher education; quality management; social control; university governance; Clark-Engwall-Soto model 


\section{INTRODUCCIÓN}

Cuatro elementos han cambiado radicalmente los sistemas universitarios en el siglo XXI, especialmente en América Latina. Primero la expansión de los sistemas con la participación de las instituciones privadas y el consecuente aumento de cobertura (Schwartzman, 2020); en segundo lugar por los cambios en la velocidad y forma de generar y circular el conocimiento científico, incluida la ciencia abierta; en tercer lugar por la instalación masiva de la enseñanza no presencial que hace difusas las fronteras geográficas; y en cuarto lugar el empoderamiento de una sociedad civil (Johnsen, 2019), donde ciudadanos escudriñadores que mediante sus trinos, blogs y periodismo ciudadano, influencian los mercados, así como a las propias Instituciones de Educación Superior - IES (Engwall, 2020).

En ese contexto de cambios, hoy más que nunca, las Instituciones de Educación Superior (IES) deben asegurar la calidad en todos sus procesos. En muchos países el Estado-Regulador, entiende su función de garante de la calidad monitoreando a las IES mediante un creciente número de registros y controles públicos y sometidas a evaluaciones que incluyen ejercicios de licenciamiento, acreditación, alimentación de bases de datos oficiales y sitios dedicados a la transparencia de las IES (Brooks et al., 2020). De modo paralelo, parece ser una tendencia internacional, que los entes reguladores delegan una parte del control sobre la gobernanza de las universidades a los actores del mercado y a los ciudadanos que escudriñan ejerciendo un control social activo (Engwall, 2020). De esta manera, los Objetivos de Desarrollo Sustentable de Naciones Unidas, las declaraciones internacionales como Budapest y DORA, los manuales como Frascati y Oslo de la OECD, los códigos éticos como los propuestos por COPE, y las listas negras internacionales de infractores como Retraction Watch Database, entre otras relevantes, se han vuelto cada vez más significativas para las universidades, toda vez que se vuelven estándares de facto que ponen en explícito las pautas de conducta comúnmente aceptadas, así como las no aceptadas.

Tradicionalmente, en la gobernanza de las universidades se han identificado dos actores relevantes ajenos a las IES: los agentes reguladores y los actores del mercado. Clark observó las tensiones entre la empresa y las disciplinas y a nivel de sistema entre la autoridad estatal-reguladora, el mercado y la oligarquía académica. También, a partir de este modelo, se han analizado las diferentes estrategias de desarrollo seguidas por sistemas universitarios (Clark, 1983). En la actualidad surge un tercer actor, la sociedad civil, que audita constantemente a las IES, asumiendo el rol de escudriñadores (evalúan, verifican, y comunican disconformidades) (Engwall, 2020).

Son abundantes los trabajos que han analizado los instrumentos y mecanismos de articulación entre las IES y el Estado-Regulador que propenden al aseguramiento de la calidad de las universidades y el sistema en su conjunto (Clarck, 1983; Donaldson y Davis, 1991; Engwall, 2020). También existen estudios que analizan los mecanismos que relacionan las IES y los mercados (Engwall, 2020; Schwartzman, 2020). Son más escasos los trabajos que analizan la relación entre las IES y la sociedad civil (Bosi et al., 2016). En todo caso, no se han encontrado estudios que hagan un levantamiento amplio de los mecanismos de articulación y aseguramiento de la calidad que surgen de la relación de las IES con el Estado, con los mercados y con la sociedad civil.

El objetivo de este trabajo es identificar y analizar los instrumentos y mecanismos de articulación de las universidades con el Estado-Regulador, los mercados y la sociedad civil; que permitirían el incremento de la calidad tanto de las universidades como de los sistemas de educación superior. Se propone una taxonomía de instrumentos organizados entorno a una versión actualizada del Triángulo de Clark (1983), la que se complementa con la Teoría de la Agencia de Donaldson (1991). Dependiendo del grado de eficacia en la implementación que las Universidades hagan de estos mecanismos e instrumentos, y la capacidad de comunicar que tengan, las IES no sólo podrán dar cuenta a los diferentes grupos de interés del nivel de calidad que las instituciones alcanzan, sino también influir en la construcción de la reputación institucional.

\section{OTROS ANTECEDENTES}

Hay una serie de antecedentes adicionales que es necesario detallar para documentar en mejor forma este trabajo: i) Modelo de Clark-Engwall-Soto; ii) Teoría de la Agencia; y iii) Aseguramiento de la calidad.

\section{Modelo Clark-Engwall-Soto}

Al considerar la aplicabilidad del Modelo de Clark, también conocido como el Triángulo de Clark a una comprensión de los sistemas de educación superior de hoy (Fan, 2018), se puede notar un debilitamiento de los límites de las IES. Podría decirse que se ha producido una menor distinción organizativa de las universidades y una invasión,-_por una parte-, por el lenguaje y las ideas del mundo empresarial (Zhou, 2021), y por otra, por los movimientos sociales, en algunos casos, distanciados de las oligarquías académicas 0 estudiantiles (Bosi et al., 2016). La ampliación de las funciones sociales en los sistemas modernos de educación superior, puede ser una de las razones por las que la autoridad académica parece estar sujeta a mayores desafíos externos. Sin embargo, gran parte del modelo analítico de Clark sigue siendo muy relevante 
para la comprensión de los sistemas e IES, incluso si algunas de sus manifestaciones empíricas han cambiado a lo largo de los años (Schwartzman, 2020).

Proponemos la actualización del modelo de Clark, sumando a los componentes: Estado-Regulador y Actores de los Mercados, a la Sociedad Civil, situando a la Universidad con sus oligarquías académicas en el centro del triángulo, el que denominaremos Modelo Clark-Engwall-Soto para el aseguramiento de calidad de las IES (ver Figura 1). En este modelo también se declaran los mecanismos e instrumentos de articulación entre los diferentes componentes que propenden al aseguramiento de la calidad.

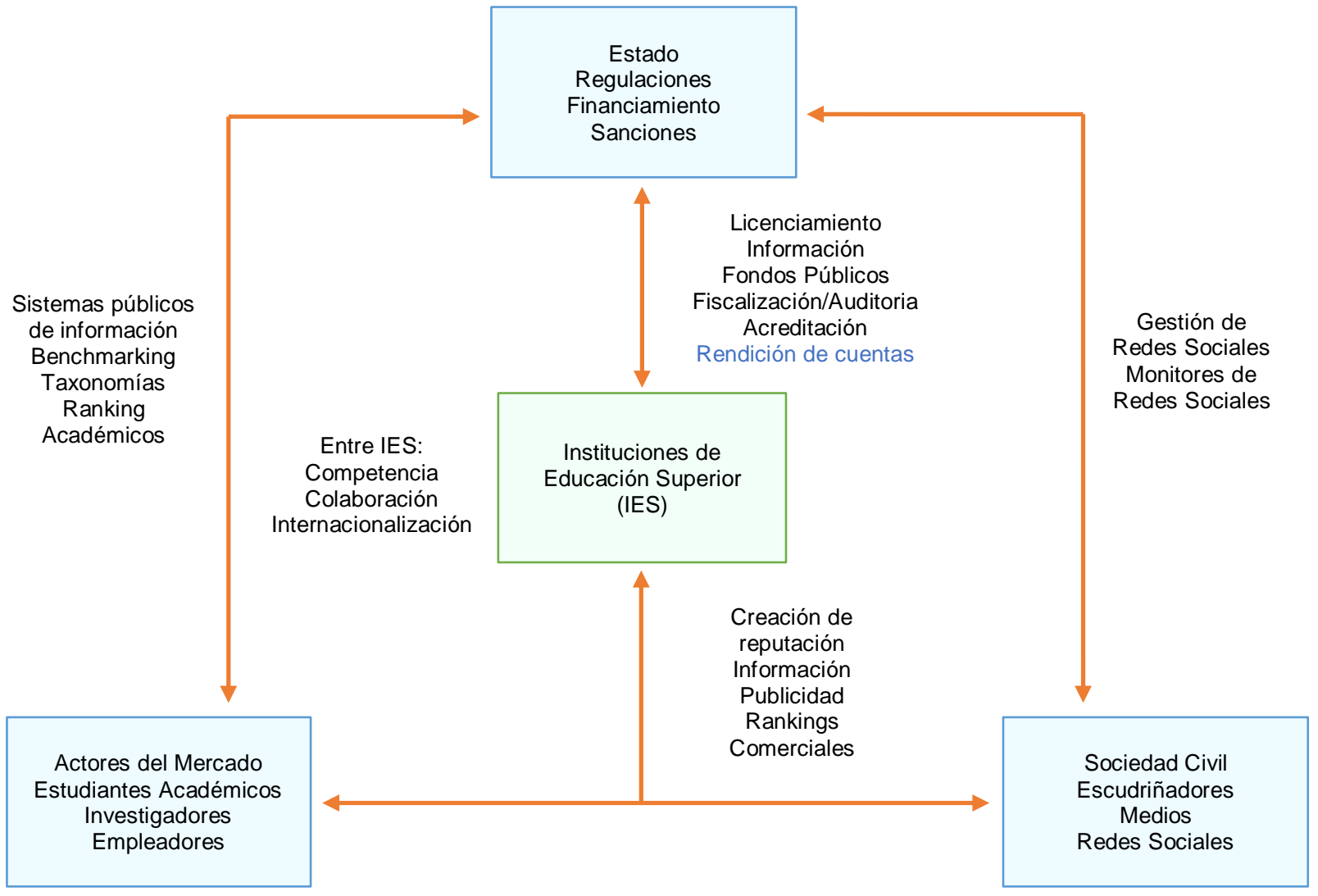

Fig. 1. Modelo Clark-Engwall-Soto para el aseguramiento de calidad de las IES. Adaptada de Clark (1983) y Engwall (2020).

\section{Teoría de la Agencia}

La Teoría de la Agencia que nace en la economía, tiene plena aplicación en las universidades públicas y privadas, con independencia del tipo de economía del país en que estas se encuentran situadas (Muga, 1997; Ganga-Contreras et al, 2015). Tanto las universidades públicas como las privadas, en general se clasifican en la tipología jurídica y tributaria de corporaciones sin fines de lucro, financiándose con los aportes del Estado (directos o indirectos) y los ingresos que generan, especialmente por derechos de matrícula (Lemiatre, 2017). El estado ha ido modificando su rol de asignar recursos al sector educación superior, privilegiando mecanismos que le permitan configurar una relación más estrecha entre financiación y el desempeño (Muga, 2001). Eso condiciona que la gestión de las IES se enmarque dentro de algunas restricciones financieras, generalmente apremiantes, sea por la necesidad de ajustar sus operaciones e inversiones al nivel de financiamiento que es capaz de producir o captar; o por su capacidad de responder a las exigencias de los entes reguladores locales, nacionales o internacionales; así como a la enorme capacidad de generar proyectos, que diversos grupos de la comunidad educativa consideran que deben ser impulsados (Lemiatre, 2017; Brunner, 2019, Schwartzman, 2020).

La Teoría de la Agencia aporta un conjunto de elementos útiles para comprender el fenómeno anteriormente descrito, especialmente si se considera al Estado como Agencia Central, y las universidades, especialmente las públicas, como agencias individuales. En la visión de esta teoría se asume que los hombres (académicos, investigadores, unidades académicas, IES) maniobramos en busca de nuestro propio interés, racionales, oportunistas y adquisitivos. Esta racionalidad está limitada por la falta de información o conocimiento respecto de cómo maximizar su conducta económica. El problema central de la Teoría de la Agencia, es asegurarse que el agente trabaje por el objetivo principal de la agencia central, cuando éste entra en conflicto con su propio beneficio. El interés principal de la Teoría de la Agencia es minimizar el costo de la agencia central. 
Desde la Teoría de la Agencia, aun cuando el Estado no intervenga en la financiación total de las IES, se asume que debe velar por la calidad de las instituciones, tanto por garantizar la eficacia y la fe pública en el sistema y sus actores, como por las consecuencias económicas que los fallos tienen para el propio Estado. Además de la natural competencia por recursos, donde las instituciones más antiguas y grandes han establecido una cierta cuota de recursos que les corresponden, impidiendo el crecimiento de las instituciones pequeñas o distantes de los centros de poder, es posible argumentar que desde un punto de vista de los entes reguladores son altos los costos de: 1) financiar la creación de una universidad pública donde no existe ninguna, versus permitir que lo haga un privado; 2) financiar la creación de carreras donde la oferta de profesionales es insuficiente para cubrir las necesidades de una región o de un país; 3) es oneroso que una IES zozobre económicamente, por el costo público de subsidiar la relocalización de los estudiantes en otras IES; 4) es un costo hundido elevado para el Estado que IES impartan títulos de baja calidad y que el mercado laboral no absorba dichos graduados.

Sugerimos, que bajo la mirada de la Teoría de la Agencia, el Estado en su condición de agencia central, ejerza su rol mediante el establecimiento de regulaciones (condiciones mínimas de licenciamiento, acreditación institucional y de programas, reconocimiento de instituciones, auditorías), el financiamiento (directo, voucher al alumno, financiamiento condicionado por proyectos, por contrato programas, por convenios marco y rendición de cuentas, entre otros) y sanciones (multas, suspensión de matrícula de primer año, pérdida de acreditación, intervención y cierre, entre otras).

A su vez, las IES deben responder a los diversos controles de la Agencia Central reguladora, incluida la provisión de información oportuna y la rendición de cuentas en todas sus formas (Cuenta Anual; Informe de Avance de Plan Estratégico; Cumplimiento del Plan de Mejora; Rendición de Cuentas al Estado y al máximo órgano colegiado de gobierno, a la comunidad a través de: Memoria Anual, Cuenta Pública del Rector, Informes de Responsabilidad Social Universitaria). El Estado, los mercados y la sociedad ponen a las IES en tensión, con base en la cual cada actor construye su reputación institucional. Las universidades entre sí, a su vez, compiten y colaboran con otras IES nacionales o internacionales, buscando generar ventajas competitivas, un mejor posicionamiento para responder al desafío de ser un actor global, campeón nacional y una casa reputada a nivel local (Engwall, 2020) (ver Figura 1).

\section{Aseguramiento de la calidad}

El aseguramiento de la calidad tiene para los entes reguladores y agencias centrales varios propósitos posibles: establecer criterios o estándares mínimos de operación para institución o programa (licenciamiento); verificar el cumplimiento de estándares de formación en diferentes campos profesionales; valorar la calidad de los claustros académicos de los programas de pre y postgrado; verificar la integridad de la investigación, y las buenas prácticas bioéticas y de edición científica; gestión y sustentabilidad financiera de las instituciones; provisión de espacios de formación y acceso a la corriente principal de la ciencia o estimular el mejoramiento continuo de las instituciones (Schwartzman, 2020). El aseguramiento de la calidad asume un rol más amplio que solo ser un instrumento de la política pública utilizado para confirmar, por parte del Estado-Regulador, los insumos, procesos y los resultados de las IES respecto de ciertos estándares de calidad.

El aseguramiento de la calidad se instala como un esquema de control distribuido, donde el Estado, las IES, los mercados relevantes, y la Sociedad Civil interactúan para crear un espacio de gobernanza en el cual convergen actores, instrumentos y procedimientos que operan en diferentes niveles y modalidades. El Estado mantiene su rol de regulación y control, situándose en la cima del triángulo, incidiendo de arriba hacia abajo: hacia las IES, hacia los mercados y la sociedad civil. En dirección a las IES, actúa mediante el ejercicio de la autoridad (obliga, permite o prohíbe), del poder de la financiación (asignación de recursos mediante transferencias, contratos, bonos e incentivos), el uso de sistemas de provisión de información, y el conjunto de medios operacionales que posee el Estado para afectar la conducta de los ciudadanos, sus propiedades o circunstancias. En contraposición, las IES - cualquiera sea su naturaleza jurídica, deben rendir cuenta ante el Estado-Regulador mediante un sistema de rendición de cuentas cada vez más oneroso, sofisticado y amplio (Lemaitre, 2017; Brunner, 2019).

En la actualidad la tendencia reguladora de los gobiernos se orienta a tener menos reglas y delega el resultado en la gobernanza universitaria, en los actores del mercado y en los escudriñadores de la sociedad civil. Por esta vía el benchmark y las normas han incrementado significativamente para las universidades, por lo tanto, es el momento que las universidades deben comunicar de forma eficaz lo que es su misión para la comprensión de la audiencia interna y externa, los rankings universitarios facilitan esta tarea de benchmark, donde la elección de los indicadores es crítica (Rider et al., 2020). Como también lo es la facilidad de acceso, interpretación y utilización en general de los sistemas de información por parte de los oficiales a cargo de los entes reguladores, actores del mercado y ciudadanos empoderados que escudriñan a las IES. Paralelamente, las propias universidades generan y despliegan acciones efectivas para gestionar la calidad, comunicar sus resultados y a partir de ello generar reputación institucional. 


\section{METODOLOGIA}

A partir del Modelo de Clark se hace una revisión bibliográfica en Scopus para identificar las políticas públicas, mecanismos e instrumentos que permiten analizar la articulación y especialmente la gestión de la calidad entre las IES, los Estados, Mercados y Sociedad Civil. La forma de presentar los resultados de este estudio, es desde una perspectiva teórica e independiente de los dominios nacionales, realizando un catastro analítico de políticas públicas, instrumentos y mecanismos que permiten estudiar la forma en que los Estados, Mercados y Sociedad Civil se articulan con las IES, e influyen en su gobernanza y gestión de la calidad.

Para la articulación Estado-IES se analizaron las legislaciones, y mecanismos de aseguramiento de la calidad generados por los Ministerios de Educación y agencias públicas como lo son las Comisiones Nacionales de Acreditación de Chile, y el Consejo Nacional de Acreditación de Colombia (Chile, Ley 21091 de 2018; CNAChile, 2019; CNA-Chile, 2020; CNA-Colombia, 2020; Colombia, Ley 30 de 1992). Para el análisis de la articulación de los Mercados e IES se analizaron los instrumentos existentes en los mismos países, los que fueron complementados con entrevistas a rectores, presidentes de comisiones de acreditación, académicos que han actuado como pares, responsables de oficinas de análisis institucional y de aseguramiento de calidad. En el caso de los rankings además de una aproximación desde las metodologías de SClmago, THE y QS, se realizaron entrevistas con especialistas de las casas de ranking. La dimensión sociedad civil-universidad, además de revisar estudios internacionales, requirió hacer un análisis de las tensiones que hoy experimentan los sistemas educativos de américa latina, lo cual incluyo la revisión de bibliografía publicadas en fuentes indexadas en Scopus, con información de prensa, y noticias publicadas en observatorios de la educación superior de diversos países de América Latina.

\section{RESULTADOS}

Para cada una de las relaciones relevantes propuesta en el Modelo Clark-Engwall-Soto para el aseguramiento de la calidad de la IES, se identifican los principales instrumentos y mecanismos, los que se muestran de forma integrada en la Figura 1.

\section{Relación IES - Estado-Regulador}

La noción tradicional del Estado-Regulador que, a través del imperio de la ley, de los instrumentos de políticas públicas y de la burocracia, determinan el comportamiento de instituciones públicas y privadas ha perdido vigencia frente a una nueva gobernanza, especialmente en las IES, que da lugar a esquemas de gobierno de múltiples niveles, con la activa participación de actores públicos y privados. Los estados promueven el uso de una diversidad de instrumentos de política, taxonomías e intercambios cómo son la competencia, información, incentivos, deliberaciones, confianza, flexibilidad y redes (Schwartzman, 2020). Los entes reguladores pueden adoptar tres formas isomorfas: la coercitiva, la mimética y la normativa. Entre los instrumentos de política pública que regulan la acción de las IES, están: el licenciamiento; el suministro normado de datos para nutrir los sistemas de información pública; el acceso a los fondos públicos; la fiscalización y auditoría; la acreditación institucional y de programas; y la rendición de cuentas; los que a continuación son analizados.

Licenciamiento o condiciones iniciales de funcionamiento, es la autorización de un ente público para que una IES abra sus puertas y reciba alumnos. En la mayoría de los países, una vez que la institución consigue esta condición, se considera autónoma y pasa a rendir cuenta a los entes reguladores mediante otros mecanismos como lo son la acreditación institucional y de programas. El fin de la valoración de condiciones iniciales es conformar una percepción preliminar de las capacidades y funcionamiento integral de la institución, para lo cual se analizan diversos aspectos, como lo son el cumplimiento de la ley, el tipo de gobierno corporativo, la organización académica y administrativa, el claustro de profesores, los recursos físicos, tecnológicos y financieros, y los principales indicadores curriculares, formativos, de producción científica, y de generación de bienes culturales (Rider et al., 2020). En términos prácticos esta es una barrera de entrada de nuevos actores a los sistemas universitarios.

La información factual y documental que es suministrada al Estado por las IES abarcan datos de: matrícula y deserción; dotación docente; espacios y recursos para la enseñanza; procesos de evaluación y planes de mejoramiento institucional en sedes y programas; y stock de investigadores (grupos de investigación e institutos). Los sistemas más avanzados ofrecen un conjunto de indicadores que proporcionan una mirada cualitativa y cuantitativa, con algunos indicadores que son sensibles a los tamaños institucionales y otros independientes de los mismos. Un sistema de información eficaz, debiera buscar, independientemente del grado de formación de los ciudadanos y de los actores de los mercados, el mayor grado de simetría de información entre los diversos grupos de interés. Uno de los roles tanto de la Agencia Central como de las universidades, es educar a la ciudadanía en la adecuada interpretación de los sistemas de información. 
Financiación, y en especial en la que la fuente es el Estado, se entiende como la concurrencia pública en financiar bienes públicos como los son la educación superior y la investigación. Los agentes ejecutores de ese financiamiento pueden ser IES reconocidas de carácter público o privado. El Estado-Regulador dispone de un amplio abanico de instrumentos para normar, monitorear, supervisar y fiscalizar de las IES la ejecución del gasto (Schwartzman, 2020). Respecto de las IES estatales, la función de control de legalidad corresponde al órgano contralor del sector público del país que tiene la función de fiscalizar el uso de los fondos públicos y verificar que las actuaciones de estas IES se ajusten a derecho. La misma facultad se aplica a las IES privadas con respecto a la ejecución de fondos provenientes del erario nacional. De esta forma se genera en los países un mercado de fondos públicos. Existe por tanto en materia de financiación pública una cuota importante de gestión y de competencia por unos recursos escasos. No obstante, en los esfuerzos por mejorar la asignación y ejecución de fondos públicos, se mantiene una relación entre las oligarquías académicas descritas por Clark y el poder político, lo cual se entiende como un rasgo de la democracia.

La financiación es indispensable para poder actuar en el mejoramiento de la calidad de las instituciones y programas. La necesidad de recursos enfocados a obtener el mejoramiento de la calidad académica a través de: reclutamiento y capacitación de docentes; la realización de investigación científica y tecnológica de calidad internacional; el desarrollo de la infraestructura física y tecnológica, de laboratorios, de acceso a recursos de información; el fortalecimiento del capital social por la vía de la internacionalización y la profesionalización de la gobernanza, entre otras estrategias. También se requiere el compromiso de las IES en la mejora de la gestión docente, de las tecnologías y financiero, tanto en la utilización eficiente de los recursos propios, así como aquellas fuentes alternativas de los distintos mercados y sectores de la sociedad. La articulación entre apertura a un mercado de estudiantes, aporte estatal en forma de voucher y condición de acreditación, presiona a las IES para avanzar en ese proceso.

Fiscalización y auditoría son mecanismos contables para asegurar que los fondos fueron utilizados para el fin asignado, mediante una selección competitiva de los proveedores y la elección - de acuerdo a unos criterios previamente conocidos con una ponderación determinada-, de la forma que los demandantes pueden obtener los bienes y servicios requeridos de una calidad adecuada (Rider et al., 2020). En el mundo académico y científico no siempre existen bienes y servicios sustitutos, en muchos casos solo existe un proveedor único. Esto no es fácil de entender por los mecanismos públicos de auditoría. Una dimensión más importante aún, es la fiscalización no solo de la ejecución financiera, sino de los resultados e impactos de los recursos transferidos a las IES mediante procedimientos condicionados, como lo son los fondos concursables (v.g. para proyectos de investigación), los convenios de desempeño, contrato programa, programas marco o los proyectos especiales.

La acreditación consiste en el análisis de los mecanismos existentes al interior de las IES autónomas para asegurar su calidad, considerando tanto la existencia de dichos mecanismos, como su aplicación y resultados sobre la calidad de sus programas académicos, su capacidad de investigar, su organización y funcionamiento y el cumplimiento de su función social. La acreditación se asume como una acción de carácter temporal, que puede ser solicitada, generalmente, de manera voluntaria por las IES. La acreditación institucional certifica el cumplimiento del proyecto de la institución, y la existencia, aplicación y resultados de mecanismos eficaces de autorregulación y de aseguramiento de la calidad (Schwartzman, 2020). Un indicador del grado de madurez respecto de la calidad de los sistemas de educación superior, es el nivel de cobertura de instituciones y estudiantes que se han sometido a procesos de acreditación.

Las sanciones son uno de los mecanismos mediante los cuales el Estado-Regulador asegura que las IES operen dentro de un marco mínimo de calidad, ellas incluyen la amonestación por escrito, las multas (especialmente por no entregar información oportuna y consistente), el congelamiento de la matrícula de nuevos estudiantes o la suspensión del financiamiento público, el cobro de garantías reales, la no renovación de condiciones iniciales, la denegación de registros calificados de programas, la no otorgación o perdida de la acreditación, y en casos extremos la intervención de las instituciones y el nombramiento de un administrador provisional con un rol similar al de un síndico de quiebras, cuyo recurso final es el cierre definitivo de una institución. En el caso de las instituciones públicas, además, aplica el conjunto de medidas que pueden tomar los órganos contralores nacionales. Las sanciones tienen un efecto directo sobre las instituciones, pero también sobre el control social, en la medida que la existencia de ellas se vuelve de dominio público, actuando de este modo sobre los mercados y sobre la sociedad civil.

Visto desde el Modelo Clark-Engwall-Soto, el aseguramiento de la calidad adquiere una fisonomía más compleja, con diversos niveles e instrumentos de acción. En él participan los diversos sectores interesados. Las IES situadas en el centro, donde en su relación con el Estado-Regulador puede sumar o restar calidad y eficiencia a las organizaciones. Las IES por su parte se diferencian entre si, gracias a la instauración en algunas de ellas de innovadores modelos de gobernanza. En un ambiente donde muchos competidores tienden a imitar a las instituciones líderes, la implementación de iguales estrategias, genera resultados muy diferentes. 


\section{Relación IES - Mercados}

Los mercados en los que participan las IES, están conformados por actores, que compiten por la demanda y suministro de bienes y servicios, ofreciendo parámetros claves como los precios y estándares de calidad (Engwall, 2020). Para efectos de este análisis, los mercados más relevantes son: el de estudiantes; el de empleos académicos; el de prestigio institucional; el mercado de capitales privados y fiscales para financiar a las IES; el laboral de los egresados; y el mercado de la innovación generada en la IES. Las IES son más o menos sensibles a los mercados dependiendo del origen de su financiamiento, y de forma especial al mercado de estudiantes, donde concurren las vacantes ofrecidas por las organizaciones y las preferencias individuales, entendida esta como expresión de la demanda (Schwartzman, 2020). En la cultura académica la sola mención de esta relación IES-Mercados produce reacciones; sin embargo, este problema valórico se contrapone al empleo del corpus de conocimiento científico generado en disciplinas como economía, finanzas o administración, aplicado a la gobernanza universitaria y la gestión de la calidad. En la Universidad transferir y aplicar el conocimiento científico existente es un imperativo categórico y un acto de coherencia.

Las IES, en su relación con el mercado, aportan información en su propio sitio web (vacantes, aranceles, planes de estudio y duración de los mismos, composición del cuerpo de profesores, nivel de empleabilidad al egresar y renta esperada), realizan publicidad, comparten testimonios, muestran evidencia de su reputación (profesores emblemáticos, presencia en rankings académicos y comerciales) o muestran sus instalaciones, aportan información a sistemas privados de información (número especiales de periódicos o semanarios), publican informes y reportes sobre su gestión (planes estratégicos, cuenta anual de rectoría, informes de responsabilidad social universitaria), y despliegan estrategias de marketing visitando los colegios de donde provienen los alumnos que luego se matriculan en ellas. Si bien el sitio web de las universidades es su mayor instrumento de relación con el mercado, en muchos casos el sitio web no refleja lo que la IES es. En el mundo actual, con pandemia, solo existen dos posibilidades: ser digital o no ser.

Información. Para el adecuado funcionamiento de los diferentes mercados, los oferentes y los demandantes requieren acceder a información de calidad, encontrarse, mostrar su atributos, capacidades y resultados. Los sistemas de información son necesarios para que un estudiante elija donde estudiar, compare los precios y las reputaciones de una misma carrera en diferentes IES; un profesor internacional o nacional postule a una plaza en cualquier institución; para que las instituciones conozcan las fuentes de financiamiento concursables nacionales o internacionales disponibles; conocer la demanda por los egresados en el mercado laboral; analizar la capacidad de comercializar patentes, emprender spin-off o comercializar la innovación generada en las IES. El Estado no puede actuar en todos los mercados, pero a lo menos debiera proveer información a los nuevos estudiantes, tanto en términos de condiciones de acceso, financiamiento de estudios y datos del mercado laboral.

Publicidad. La publicidad, que generalmente forma parte de un plan de comunicaciones institucional, tiene como fin anunciar la oferta, mostrar fortalezas y educar a la demanda en la forma de escoger su mejor opción (Vetrova et al., 2019). En este sentido, las instituciones públicas están limitadas a realizar publicidad de carácter informativo. Un aspecto particular, pero relevante, es que una institución o un programa académico de educación superior solo podrá utilizar el calificativo de acreditado cuando el órgano competente se lo otorgue.El plan de comunicaciones abarca la combinación de medios utilizado por cada IES, mediante los cuales buscan llegar a diferentes audiencias, especialmente a los jóvenes con potencial interés. Las universidades buscan generar presencia referida a la visibilidad web, la reputación institucional y la presencia de la Universidad en el entorno digital, impulsando la puesta en valor y apropiación social de los servicios académicos, de investigación y de proyección social (Vincent et al., 2020). Junto al diseño y administración de los sitios web se encuentra el desafío de la gestión de las redes sociales, generalmente a cargo de un equipo de Community Manager.

Las IES son activas definiendo y consolidando los canales digitales que les permitirán ofrecer contenido académico valioso para las audiencias deseadas: clases en abierto, datos científicos en abierto, documentos científicos en abierto, colecciones patrimoniales en abierto; mediante los cuales las IES regresan a la sociedad lo que hace, lo que son, y los bienes culturales que la propia sociedad les brindo. Para que esta presencia en lo digital ocurra de un modo adecuado y eficaz parece ser deseable la existencia de una cultura organizacional y de una gobernanza, que permita mostrar de forma eficaz a diferentes grupos de interés, como la IES cumple su misión, proyecto académico y aporta valor a la sociedad.

Rankings universitarios académicos y comerciales. Las universidades buscan a través de rankings comunicar como las casas de ranking internacionales o nacionales las ordenan con arreglo a un conjunto de indicadores que buscan por la vía de la comparación situar a las universidades en determinadas posiciones en relación a las otras. Los rankings se han construido con base en metodologías que miden el desempeño de una casa de estudios superiores en relación con su función docente y de investigación, y con menor intensidad los impactos societales. Los rankings de IES enfrentan el desafío de ordenar instituciones con diferentes misiones, historia, proyectos educativos, tamaño, oferta académica de pre y post grado, capacidades 
científicas, recursos, estatutos, control político-administrativo, y mecanismos de aseguramiento de la calidad (Bustos-González, 2019).

Los rankings fueron inicialmente concebidos como un instrumento que permite medir la eficacia de una IES, asumiendo que una universidad altamente ranqueada es más productiva, imparte docencia de alta calidad, realiza investigación de alto impacto, y hace una mayor contribución a la sociedad que las instituciones ranqueadas por debajo de ella. Sin embargo, el fortalecimiento de una de las áreas esenciales, como son docencia, investigación y proyección social, pudieran generar un conflicto con el adecuado despliegue de las otras. Esta realidad pone de relieve la necesidad, al menos teórica, de medir cuándo se está en presencia de una institución de educación superior que alcanza la calidad. La estrecha relación entre calidad de la enseñanza e investigación ha sido ampliamente discutida, analizada y demostrada a través de los trabajos de Geschwind y Broström, 2015; Shu-Ling-Huang y Shen, 2017.

Es difícil encontrar buenos indicadores de la calidad del aprendizaje, de la investigación y de la proyección social que representen los verdaderos resultados de las universidades, y más si esta medición es de escala global. Los rankings realizan un esfuerzo para objetivar variables cualitativas mediante la identificación de indicadores cuantitativos que mejor representen las universidades que alcanzan la calidad (Qureshi et al., 2021). No todos los rankings se basan en información objetiva. Algunos generan indicadores a partir de encuestas de opinión.

Los rankings universitarios se pueden clasificar básicamente como globales o nacionales. Adicionalmente, distinguimos entre los rankings académicos creados con base en información objetiva recogida de fuentes independientes de la universidades ranqueadas, tal es el caso del SClmago Institutions Ranking (Scimagoir.com), cuyas fuentes de datos son Scopus, Europena Patent Office, Google, Ahrefs, Mendelay y PlumX; y el Ranking Web of Universities (Webometrics.info), cuyas fuentes de datos son Google, Majestics, Ahrefs y SCImago; de los rankings híbridos o comerciales, donde solo una parte de los datos proviene de fuentes independientes y otra es solicitada a las propias universidades. Generalmente, estos rankings comerciales proveen por suscripción servicios adicionales a las universidades, tal es el caso de THE, QS, y en general de los rankings nacionales.

En la relación IES-Mercado, los rankings aportan una visión independiente de las IES respecto de su posicionamiento en relación a otros competidores. Los rankings académicos cumplen esta función de mejor manera que los rankings comerciales, porque los primeros carecen de conflictos de interés. Otro riesgo importante es que los indicadores sensibles a los tamaños institucionales hacen que las universidades más grandes de cada país tiendan a ocupar las primeras posiciones, generando una distorsión de la mirada cualitativa de la IES. Cuando los rankings son utilizados en la publicidad de las Universidades no se advierte sobre este riesgo.

Accountability o rendición de cuentas. Hoy para muchas universidades del mundo resulta indispensable la evaluación y la rendición de cuentas pública. Las IES cada vez son más activas en informar a la comunidad universitaria, a las entidades financiadoras y a la sociedad en general, sobre los procedimientos y resultados de la evaluación y sobre los niveles de calidad alcanzados. Esta transparencia permite concurrir con información confiable a los procesos y convocatorias competitivas. Muchas universidades consideran la rendición de cuentas un deber ético, con el fin de responder e informar de manera periódica, planeada, pública y participativa sobre: la realización de la misión y el proyecto educativo institucional, la adecuada gestión, el eficiente manejo de los recursos, el avance y los impactos de los proyectos, las expectativas cumplidas y no cumplidas y en general, de los resultados de la Institución, permitiendo analizar la evolución intertemporal de las dimensiones analizadas, así como las comparaciones con el contexto nacional o internacional cuando ello sea posible (Dandala, 2020). La rendición de cuentas no debe solo ser un acto performático, los soportes audiovisuales y documentales deben quedar de dominio público de forma permanente, lo cual ayuda a generar memoria organizacional, a los escudriñadores a acceder a fuentes confiables y a construir la reputación de la institución.

Marketing científico, es el conjunto de acciones que una universidad, un instituto, grupo de investigación o investigador individual desarrolla para hacer visible su producción científica. El marketing científico ayuda a generar visibilidad y de esa forma impacto científico sobre un conjunto de agregados, como lo son la comunidad académica y de modo muy directo sobre sus estudiantes; sobre el medio ambiente incluido los egresados, las empresas, y la sociedad; sobre los actores públicos y las leyes y políticas que impulsan; sobre la calidad de vida de las personas y el cuidado del medio ambiente. Estas acciones de visibilidad incluyen un conjunto de estrategias para poner los productos de la actividad científica y académica en contacto con diversas audiencias, para lo cual se utilizan diferentes plataformas de redes de comunicación científica, académica y social.

Creación de reputación. El capital de la universidad es su prestigio. Este se puede fundar en un acervo reconocido por la sociedad o ser construido a través de marketing. Para la creación de reputación, las IES 
recurren a la información, la publicidad y la presencia en los rankings, sean éstos de carácter comercial o académicos. En su relación con los mercados, las IES fomentan su presencia en sistemas públicos de información, participan en benchmarck o los generan ellas mismas. Por su parte, en la relación de las IES con los escudriñadores (agentes sociales), éstos aportan datos a los sistemas de información privados y adoptan buenas prácticas, generalmente internacionales, para intentar alinear la conducta de sus miembros con las pautas de conducta y desempeño esperadas por la sociedad.

Los estados de opinión respecto de las IES se construyen con base en atributos subjetivos (Bustos-González, 2019). Los distintos actores de la sociedad aplican una percepción selectiva de la realidad, lo que genera de manera agregada una noción de reputación, que como es percibido, en muchos casos está sólidamente ganada y en otros no coindice con la realidad objetiva. La lógica de la reputación, entendida como calidad percibida, es inclusiva y cooperativa, mientras que la de los rankings puede resultar excluyente y competitiva. Todas las universidades, independientemente de su presencia en los rankings y de sus tamaños relativos, aspiran a gozar de una excelente reputación. Dependiendo del público objetivo la noción de reputación se construye sobre unos criterios variados. Si bien la reputación se puede objetivar por medio de rankings académicos, estos no son, la única fuente de información utilizada por los grupos de interés para posicionar instituciones (Maduro et al., 2018).

Los estudiantes son un agente clave en la construcción de reputación institucional. Para los estudiantes, la reputación de la universidad depende sobre todo de la calidad de la docencia. Los estudiantes eligen su universidad con la expectativa de recibir una buena formación, contar con profesores expertos y cercanos, donde perciban que la universidad es activa en asegurar la calidad de la enseñanza y facilita la creación de redes que aumenta el capital social de sus egresados. Los alumnos valoran la oportunidad de estudiar y las condiciones en que lo hacen. En ellos influyen: la posibilidad de acceder a becas de estudio, alimentación, transporte, la facilidad para encontrar alojamientos adecuados, las oportunidades de desarrollar actividades deportivas, culturales, de solidaridad y protección al medio ambiente.

El nivel de compromiso (engagement) de los estudiantes con su casa de estudios se construye tanto de forma activa (clubes deportivos, ropa con logo) como por la cotidiana experiencia de la vida universitaria. Las asociaciones de antiguos alumnos son una consecuencia de la solidez de la relación que nace de una experiencia satisfactoria de los estudiantes. Si sus expectativas han sido satisfechas, si la formación recibida es percibida de calidad, si los egresados se sienten exitosos y agradecidos de su universidad, es probable que se expresen cuando ya son profesionales y decidan colaborar con su universidad. Una universidad tiene buena reputación cuando sus estudiantes y egresados tienen una buena reputación de ella (Brooks et al., 2020).

La reputación académica, es un intangible, percibido por los colegas, estudiantes, autoridades académicas, empresariales y públicas. Es la impresión general de excelencia académica y calidad humana creada por una serie de factores como lo son: los grados académicos, las capacidades docentes, los resultados de investigación, capacidad de escuchar a los estudiantes y de responder a sus preguntas, el compromiso y la participación en la gobernanza universitaria, la presencia en los medios públicos de comunicación, la membrecía en sociedades científicas, el reconocimiento y admiración de sus pares. Una institución que cuenta con un claustro académico de muchas personas de alta reputación académica, altamente comprometida con los estudiantes y la vida universitaria, genera una comunidad vibrante, inteligente, creativa e innovadora; que es valorada por la comunidad estudiantil y por la sociedad extra muros (Schwartzman, 2020).

La reputación científica, es un componente de la reputación académica. Para que una persona sea reconocida como un investigador debe reunir unos antecedentes de formación, experiencia investigadora, haber ganado financiamiento extra institucional concursable para sus proyectos (grants), generar resultados de investigación, haber recibido premios, y que su producción científica tenga impacto en la comunidad científica, académica, económica o social. La reputación científica es el nivel de prestigio que un investigador construye a partir de la opinión que la sociedad tiene respecto de su actividad investigativa. No solo se trata de tener una producción científica continua en el tiempo, sino de generar impacto (Reed, 2021).

El prestigio es un fenómeno transferencial que solo puede dar quien lo tiene. Por lo tanto, importa quién, donde, cuando y con qué frecuencia citan el trabajo de un investigador. Una institución que cuenta con un stock de investigadores activos, cuyos indicadores cualitativos muestran resultados de impacto liderado en, o por sobre la media del mundo o de excelencia liderada en los percentiles más altos, permiten inferir que los estudiantes se benefician de la experiencia de estar en contacto con personas que exploran la frontera del conocimiento, y por tanto pueden transferir a sus alumnos dicha competencia.

La reputación social de una IES, se construye a partir de la reputación percibida por los estudiantes, percibida y proyectada por sus académicos e investigadores, pero además por su presencia en la prensa, las redes sociales, la opinión de los funcionarios públicos, la opinión de los empleadores y por la opinión de la sociedad civil que las escudriña aplicando el control social. La reputación es un intangible frágil, especialmente en el 
mundo actual. La marca y el poder simbólico de las universidades, son un ingrediente principal de la información y publicidad que las instituciones entregan al público y que la autoridad busca regular a fin de evitar su utilización engañosa. A su turno, todos estos factores influyen sobre la percepción que los diferentes grupos de interés tienen de las IES y sobre la confianza que manifiestan en ellas los estudiantes, sus familias, los empleadores y la opinión pública en general.

\section{Relación IES - Sociedad Civil}

La sociedad civil está conformada por los medios de prensa, las organizaciones no gubernamentales y los individuos que hacen uso de las redes sociales. La acción combinada de estas tres tipologías de actores, que están altamente conectados y generalmente pobremente coordinados, influye sobre la gobernanza institucional, la gestión de la calidad y la reputación de las IES. La sociedad civil a través de los escudriñadores y la regulación de los mercados, proveen cánones, eso es normas tácitas y explícitas respecto de las buenas prácticas de las instituciones. Ellos mediante denuncias que castigan a las instituciones participantes en diferentes mercados, sancionan a quienes transgredan las normas (Engwall, 2020).

Las Universidades se pueden entender como comunidades discursivas que cohabitan en comunidades mayores, compuestas de ciudadanos, que, gracias a las redes sociales, pueden difundir sus ideas, experiencias y opiniones. Las Universidades viven en la sociedad, y por tanto son relevantes su capacidad de escucha, diálogo, consideración e influencia en ella. Para ello las IES incorporan en su gobernanza a representantes de diversos organismos externos, expresivos de la sociedad civil y de las comunidades locales, para coadyuvar en los procesos internos de autoevaluación y gobernanza. Esta incorporación puede ser permanente o temporal, en instancias formales o informales de la organización, en el nivel del gobierno y gestión de las IES o dentro de sus facultades, escuelas o institutos (Rider et al., 2020).

Información. La misma información que los entes reguladores y las IES ponen a disposición de los mercados, debiera estar a disposición de la sociedad civil. El desafío en ambos casos es reducir las asimetrías de información. Una característica actual de la sociedad civil, es que los flujos de información además de circular por los medios de prensa, producción académica, ensayos e informes; también fluye por redes sociales como Tweet, ResearchGate, Linkedin, Facebook, Instagram y Wikipedia. La velocidad y capacidad de expansión de las redes sociales es vertiginoso. Las universidades requieren gestionar sus comunicaciones con inteligencia y profesionalismo.

Publicidad. En el contacto con la sociedad civil, tanto la publicidad convencional como las redes sociales requieren de atención y contenido. En el marketing digital, emergen nuevos protagonistas, como es que algunos miembros de la comunidad académica adopten el rol de influenciadores (líderes de opinión), operando a través de las redes sociales. Cada institución establece un marco ético de hasta donde considera que su actuación puede llegar. Las acciones desplegadas para ganar algunos me gusta (likes) puede terminar espantando a personas de reputación política, académica, científica o empresarial, que no están dispuestos a transferir su prestigio a determinadas universidades. La respuesta de las IES ha consistido en segmentar las audiencias por canales diferentes (plataformas específicas), y diferenciar los mensajes por audiencia y canal, para reforzar mensajes específicos, y de esa forma satisfacer a la vez de la mejor las expectativas de diferentes grupos de interés (potenciales estudiantes, académicos, directivos, consejeros superiores) (Vetrova et al., 2019; Vincent et al., 2020).

Los rankings comerciales, son un aliado útil en este esfuerzo por segmentar audiencia, reducir grandes cantidades de información a un único indicador que muestre en qué posición relativa queda situada una universidad. Los rankings regionales, nacionales, por profesión o determinados estudios de postgrado (MBA), ayudan a esta segmentación. El primer escollo a subsanar es tener el tamaño mínimo necesario para que la universidad ingrese a dichos rankings (Torabian, 2019).

Rendición de cuentas. Los esfuerzos que las IES hacen de rendiciónn de cuentas, son útiles en primer lugar para su audiencia interna. El beneficio de rendir cuenta y actuar de forma transparente es mayor que el costo de no hacerlo. La sociedad exige cada vez más que las universidades sean éticas, en la relación profesoralumno, en los procesos de evaluación, en las buenas prácticas de investigación y en la edición científica. La forma en que la Universidad muestre que es proactiva en estas materias es la rendición de cuentas, la que finalmente la protege, cuando se desatan escándalos, mostrando que la institución definió políticas y tomó medidas oportunas para tratar de prevenir dichas situaciones, y por lo tanto, que finalmente estas son responsabilidad de personas individuales y no de la institución (Engwall, 2020).

El marketing científico, en relación con la sociedad debiera denominarse apropiación social del conocimiento. Este por una parte pone el conocimiento generado en la Universidad al servicio de la sociedad en su conjunto, tanto en sus productos científicos y de innovación, como en diversas formas de divulgación científica. Las IES de alguna forma son entes con credibilidad social que puede desmentir las noticias falsas sobre verdades 
científicas. A su vez, si las universidades se ven involucradas en escándalos de integridad o producción científica, esa posición de autoridad se ve socavada.

Creación de reputación institucional. En este aspecto la relación de las IES con la sociedad civil está sufriendo una transformación vertiginosa, producto de las acciones de control social y el rol jugado por los escudriñadores a través de las redes sociales. Lo que los académicos consideraban la forma natural de relacionarse con la sociedad, eso es desde el pedestal de la sabiduría, se ha visto destruido o a lo menos morigerado por las dinámicas de las redes sociales.

Parece ser que los conflictos estudiantiles responden cada vez menos a detonantes internos y más en conflictos de la sociedad, pero las consecuencias de los mismos afectan a los miembros de la comunidad educativa y a la sociedad en su conjunto, y por tanto a la reputación institucional. La resolución de los conflictos en muchas oportunidades escapa al control de las autoridades universitarias. ¿Cuál es el costo institucional de la paz estudiantil? Los mecanismos que las universidades consideraban parte del estilo académico de construir reputación institucional, como lo son: reclutar nuevos alumnos, comunicar información, formas de hacer publicidad, participar en ranking académicos y comerciales, mecanismos de rendición de cuenta, apropiación social del conocimiento, emprendimiento e innovación, pierde valor cuando los conflictos estudiantiles se alargan y tienen cobertura mediática (Christensen et al., 2020).

Elementos internos como prestigio de sus académicos, calidad de la docencia, formación extracurricular, vida universitaria, reputación científica, engagement de los estudiantes, profesores y funcionarios, paz estudiantil, nivel de la infraestructura tecnológica y de comunicaciones, calidad de las instalaciones, dotación de laboratorios, calidad de sus bibliotecas y otros apoyos al aprendizaje, visibilidad de la producción científica; sumado a elementos del entorno, como lo son acreditación institucional, presencia en rankings académicos y comerciales, acceso a financiamiento suficiente, posicionamiento web, presencia e impacto en redes sociales, acciones efectivas de rendición de cuenta, puesta en valor y apropiación social de los servicios académicos, de investigación y de proyección social son ingredientes vitales.

La reputación institucional no se limita a unos pocos indicadores de posicionamiento web gestionados por el efecto de la publicidad, el marketing digital, la contratación de influenciadores o el despliegue de una eficaz estrategia de comunicaciones. La reputación de una IES se construye a partir de la percepción de los estudiantes, de sus académicos, de la presencia en la prensa, en las redes sociales, por la opinión de los funcionarios públicos, la predisposición de los empleadores y por la opinión de la sociedad civil que ejerce el control social. La reputación es un intangible frágil.

\section{Relación Sociedad Civil - Estado}

La sociedad civil puede ser entendida como la suma de las necesidades particulares de los individuos, que buscan satisfacerlas. La suma del interés por satisfacer determinadas necesidades genera un flujo de nexos recíprocos entre los hombres, y crea un nivel específico de interacción y comunicación que se identifica como la sociedad civil. Las redes sociales sin duda ayudan a que esos individuos se encuentren, compartan ideas y puedan alcanzar un cierto nivel de articulación (Bosi et al., 2016). La sociedad civil está cada vez más empoderada, sabe que puede poner en jaque al Estado, el que para una parte de la sociedad ya no es visto como la máxima estructura que representa a la sociedad en su conjunto. Un ejemplo de ello son los conflictos sociales vividos en Chile, Colombia, Perú y Ecuador desde mediados del 2019, y en Estados Unidos a inicios del 2021. En la actualidad el mecanismo preferente de articulación de la sociedad civil son las redes sociales.

Gestión de redes sociales. Las redes sociales se han consolidado como uno de los factores fundamentales en las estrategias de las IES. Esto se debe, no solo al crecimiento exponencial de su penetración en la sociedad, sino también a sus características vinculadas a las narrativas digitales, más afines a las expectativas de los estudiantes actuales, futuros estudiantes y egresados recientes. La posibilidad de segmentar la comunicación y de recibir información de las audiencias en tiempo real, así como la reducción de costes, han hecho posible que los canales sociales desplacen a los medios tradicionales como fuente principal de información e interacción (Guerrero-Solé et al., 2020).

En el caso particular de las instituciones de educación superior, este panorama representa una oportunidad para construir relaciones de confianza con la comunidad y encontrar nuevas dinámicas para los procesos de aprendizaje, de producción de conocimiento y de difusión de estos, dando a conocer la universidad, su calidad y sus frentes de acción en diferentes escenarios fuera del contexto local y regional. Como contrapartida, los efectos de la comunicación institucional por redes sociales son temporales, efímeros: Los administradores de Ios canales sociales de las IES debe permanentemente buscar nuevas formas de transmitir los mensajes a su audiencia.

Monitoreo de las redes sociales. Las universidades monitorean las redes y analizan los riesgos de los mensajes negativos, los que buscan minimizar mediante mensajes positivos, acciones de transparencia activa y en definitiva educando a sus públicos objetivos, a la sociedad civil, neutralizando a los escrutiñadores y 
haciendo que los influenciadores generen opiniones positivas. Este es un campo de acción relativamente nuevo y polémico, en donde no todos los actores se sienten cómodos con el fondo y la forma. Pero la evidencia indicaría que su importancia es creciente y no se puede desentender. Sin embargo, cada institución puede establecer el marco ético, el público objetivo, los canales utilizados, los mensajes, el tono empleado y la línea gráfica.

\section{Relación Mercados - Estado}

La relación entre los mercados y el Estado-Regulador, de lo privado y lo público, es una de las grandes cuestiones en la concepción de los sistemas y tradición de los países, reflejado en sus constituciones y tensionado por un debate constante entre los poderes económico y el interés social. En materia de la provisión del derecho a la educación, no existe un consenso universal si el Estado debe proveer el servicio o solo garantizar el acceso o hasta que nivel educacional debe garantizar la cobertura. Además, el Estado, debe asegurar a las IES un acceso racional y un uso eficiente de los recursos que el Estado pone para la infraestructura académica, los estudios de pre y post grado, la investigación y la innovación. En todo caso, existe un cierto grado de consenso que los mercados funcionan mejor cuando hay libre competencia y un libre acceso a información confiable y oportuna (Dougherty, 2020).

En dirección a los mercados, el Estado opera con un conjunto de instrumentos similares a los empleados en la relación IES-Estado, como lo son el financiamiento a la demanda, información y comunicación pública de acreditación, resultados de auditorías y sanciones; todas estrategias desplegadas en conformidad con el objetivo general de crear, regular, informar, limitar o suprimir los mercados relevantes para la IES. Básicamente se trata de la función de regulación de las libertades del mercado que, por el lado de las organizaciones proveedores consiste en la facultad de determinar la oferta de programas, vacantes y el precio de los aranceles y; por el lado de los estudiantes, en la posibilidad de elegir, cambiarse de institución o programa, contar con la necesaria información y estar protegidos del abuso y el fraude.

Sistemas públicos de información, cubren un rol fundamental, generan simetría de información entre los actores. En tal sentido los sistemas públicos de información deben ser integrados, confiables, consistentes y estar actualizados, especialmente en fechas en que los actores del mercado deben tomar decisiones, como lo es la elección de una IES para estudiar.

Benchmarking, generalmente es una tarea entregada a los investigadores de la educación superior, a las asociaciones nacionales de universidades o consejos de rectores, y a los rankings. En la medida que un país cuenta con un conjunto de investigadores de larga trayectoria investigando sobre el desempeño, gobernanza, aseguramiento de la calidad, se contará en el país con estudios serios y actualizados, comparaciones útiles con sistemas de educación superior de otros países, y un grupo de especialistas que ayuden a construir mejores políticas e instrumentos de política pública, gestionar mejor las instituciones del estado y a apoyar a las IES en su desarrollo (Tuesta et al., 2020).

Taxonomías universitarias. Son intentos o formas en que el Estado $u$ otros analistas de los sistemas universitarios clasifican las instituciones por tipologías similares. En el caso del Estado para definir políticas públicas homogéneas para grandes conjuntos de instituciones. Para los investigadores es una forma de comprender las características del sistema, generar análisis válidos para un conjunto representativo de IES, proponer mejoras que permitan el desarrollo de la educación superior, así como para encontrar nuevas formas de incentivar, evaluar y mejorar la calidad universitaria. Las Universidades, en la mayoría de los sistemas, son uno de los actores incluidos dentro de las IES. Esto en sí mismo ya es una taxonomía de uso común, donde los nombres genéricos de las tipologías de instituciones diferentes en cada sistema nacional.

Las taxonomías son importantes, pues ellas generan una diferenciación del rol social de las distintas clases de instituciones que conforman los sistemas de educación superior de los países. Son útiles para la toma de decisiones de los futuros estudiantes a la hora de elegir una IES (Rider et al., 2020). Las categorías que componen una taxonomía y el uso que se haga de ella en los nombres de las instituciones, facilita la comprensión de los nuevos estudiantes y de sus familias de la naturaleza de la institución que están eligiendo. Cuando en los sistemas nacionales reservan el uso de la palabra universidad en el nombre de una institución que cumple con la condición taxonómica de serlo, se genera un mensaje consistente hacia los mercados, y de modo especial al mercado de nuevo estudiantes. Sin embargo, cuando las legislaciones nacionales, permite el uso de la palaba universidad o expresiones entendidas como cuasi sinónimos, en instituciones de educación superior de nivel técnico, se puede producir confusión en algunos actores de la sociedad. Los nombres de las tipologías institucionales debieran ser singularizantes y denotativas de la categoría taxonómica que el Estado asigna a esa casa de estudios.

Ranking Comerciales son un instrumento que permite a los actores del mercado entender el desempeño de las IES, y de modo especial a las universidades. Se propone diferenciar los rankings nacionales de los internacionales, entendiendo que en la relación Mercado-Estado, los nacionales incluyen a la mayor parte de 
los actores institucionales y en muchos casos presentan arreglos por las principales titulaciones (v.g. medicina, derecho, ingeniería), proveyendo a los actores del mercado de postulantes, una herramienta útil para la toma de decisiones mejor informadas. El factor crítico para valorar la utilidad de un ranking radica en la relevancia de los indicadores utilizados, en la capacidad discriminante de los mismos, y en la capacidad de reflejar los aspectos cualitativos de las instituciones y sus programas de forma independientemente de los tamaños institucionales, en la ponderación de los mismos, y en la fuente de datos con que se construyen los indicadores. Desde la perspectiva de la IES, el mejor ranking es aquel donde mi institución queda mejor posicionada.

Ranking Académicos. Ellos avanzan sobre una caracterización de las IES, especialmente las de nivel Universitario, con foco en aspectos y dimensiones medibles a nivel internacional: capacidades y resultados de investigación, composición del cuerpo de profesores, visibilidad institucional en la web y redes sociales, percepción de egresados y empleadores. Los rankings mundiales ofrecen una métrica similar para sistemas e instituciones heterogéneas. La gobernanza universitaria, la internacionalización de las IES y la construcción de reputación institucional se ven enriquecidos por un uso inteligente de estas herramientas de diagnóstico y comparación internacional.

Los rankings tienen algunas limitaciones: 1) los mundiales no son exactos y los nacionales muy limitados; 2) en algunos rankings falta independencia entre la institución ranqueada, el origen de los datos y la generación de indicadores; 3) en algunos existe un limitado grado de transparencia de las metodologías y de consistencia en el empleo de la misma metodología en los rankings de regiones geográficas respecto del mundial; 4) otros rankings tienen una baja capacidad discriminante entre las instituciones líderes respecto del conjunto de instituciones analizadas; 5) el tamaño de las instituciones pesa mucho en las posiciones de ellas en los rankings; 6) algunos rankings ponen el foco en World Class Universities y poco en universidades docentes o universidades docentes que hacen investigación; 7) algunos rankings se basan en opiniones y resultados de investigación e ignoran las otras misiones de las universidades; 8) las ponderaciones de las variables son arbitrarias, e incluso dentro del mismo ranking varían según el subconjunto de países cubiertos; 9) los rankings por lo general son de instituciones completas, por lo tanto, no necesariamente son válidos en áreas temáticas especificas (v.g. educación). 10) la percepción pública pesa en una proporción alta en la posición de las instituciones. Pese a todas estas críticas, los rankings académicos internacionales son tomados en cuenta especialmente por universidades de investigación y por las universidades docentes que hacen investigación (Bustos-González, 2019).

No cabe duda que los sistemas de información, los benchmarck, las taxonomías, los rankings académicos y comerciales, son instrumentos que hacen que los mercados tiendan a operar de forma más perfecta. Sin embargo, la evidencia muestra que el Estado-Regulador limita su actuación sobre los sistemas de información, las taxonomías, y de modo menos frecuente en la autorización de nuevas titulaciones, en el tamaño de la oferta de vacantes y en bandas de precios de las matrículas. Es altamente recomendable que los demás instrumentos funcionen de forma independiente del Estado. Parece no ser sano para un sistema de educación superior, que el Estado genere un ranking de universidades, cuando luego por el imperio de la ley, también es el responsable de acreditarlas. El Estado al generar el ranking emite un juicio público que después ya no puede contradecir a la hora de acreditar. El uso que los mercados hace de indicadores, rankings y taxonomías, variará de acuerdo a su madurez y capacidad de análisis.

\section{DISCUSION}

Este trabajo realiza un levantamiento de los mecanismos utilizados por las IES para rendir cuenta de la calidad alcanzada al Estado-Regulador, a los mercados y a la sociedad civil. El modelo propuesto, denominado ClarkEngwall-Soto, organiza los principales instrumentos de política pública y gobernanza privada utilizados para gestionar la calidad de las IES. Si bien todas las, políticas, instrumentos y mecanismos incluidos en esta taxonomía existían y son de relativamente amplia aplicación, en la literatura del área habían sido analizados individualmente (Lemiatre, 2017). El modelo Clark-Engwall-Soto los organiza en términos de función social. La integración que se presenta resulta ser valioso tanto desde la perspectiva de las IES, como del EstadoRegulador.

Se rescata el valor del Estado en su rol regulador de los sistemas nacionales de educación superior, el que, mediante políticas públicas, incentivos y controles, impulsan a que las instituciones públicas y privadas propendan a la calidad (Lemiatre, 2017). En algunos países, como lo son Colombia y Perú, el EstadoRegulador pareciera confundir las políticas e instrumentos que fomentan el aseguramiento de la calidad con la recolección de datos y generación de evidencias granulares de bajo o nulo valor para el sistema y sus actores. En el caso de Colombia, más se objetive los factores y enumeración de características en los procesos de acreditación institucional, de forma contraria a conseguir mayor objetividad, lo se logra es castigar los proyectos educativos originales, y las prácticas se vuelven homogéneas, fomentando la copia más que la diferenciación. En los casos nacionales mencionados, ni los datos, ni las evidencias, garantizan el nivel de calidad de las instituciones, o genera una mayor simetría de información entre los actores. Paradójicamente, 
tampoco asegurar la objetividad del proceso a la hora de otorgar la acreditación institucional o de programas. Coincidentemente con Muga (2001), lo argumentado anteriormente no está en contra de la necesidad de las instituciones de proveer a los sistemas nacionales de acreditación de un conjunto mínimo de datos que caracterizan diacrónicamente a las instituciones y la mejora continua de sus procesos principales.

Se sugiere que si el Estado-Regulador pusiera el foco más en los mecanismos sistemáticos y permanentes de aseguramiento de la calidad que las diferentes instituciones del sistema deben proveerse, admitiendo la diversidad en sus formas, como sucede en Chile, y menos en las evidencias particulares estandarizadas como sucede en Colombia, probablemente los sistemas tenderían a ser más eficaces en la tarea de asegurar la calidad. Es deseable que el Estado-Regulador en los diferentes países contribuya a generar en los mercados y sociedad civil la noción de que existe un costo para las IES de no estar acreditadas institucionalmente.

Coincidentemente con Muga, et al. (2019), en algunos países más avanzados, la búsqueda de la calidad de la educación superior es un rol que no solo descansa en el Estado-Regulador. Las Universidades en genuino intento de mejorar multidimensionalmente su calidad, introducen innovaciones en la gestión de sus funciones sustantivas, a través de cambios en la gobernanza, mecanismos de planeación y control de gestión y financiero, y responden a la presión de la competencia en diferentes mercados. Coincidentemente con BustosGonzález (2019), en este sentido los ránquines académicos y marginalmente los rankings comerciales, producen una suerte de detonante, donde dimensiones como investigación, docencia, innovación tecnológica y apropiación social del conocimiento se transforma en arenas donde la competencia se da, y genera movimientos en algunos casos sorprendentes. Ejemplo de ello es Chile, pasó de tener solo dos universidades de investigación en 2018 a tener 4 en 2020. O Colombia donde en el mismo indicador logra que tres universidades obtengan producciones científicas con impacto normalizado liderado sobre la media del mundo y Excelencia 10 liderada sobre el 10 por ciento esperado. En otros países, como México y Argentina, Perú y Ecuador, algunas universidades privadas se valen de rankings de ambos tipos para diferenciarse (Ranking SClmago, 2020).

Por su parte, las IES en su articulación con el Estado, los mercados y la sociedad civil pueden ser activas tanto en medidas que aseguren la calidad como en acciones que informen a diferentes grupos de interés de la calidad alcanzada. Sin embargo, respecto del nivel de logro de las IES, también existen mecanismos de articulación entre los mercados y la sociedad civil, de la sociedad civil respecto de las IES y de los mercados respecto de las IES. Esta red de grandes grupos de interés tensiona a las IES y genera diferenciaciones entre las instituciones que componen los sistemas nacionales, y genera atracción hacia instituciones que situadas fuera del país.

Un aporte de este trabajo es que a partir de una versión modernizada del Triángulo de Clark (1983) y el empleo de la Teoría de la Agencia de Donaldson (1991), se identifican los principales instrumentos y mecanismos de interacción entre los componentes antes indicados. En la línea de lo aportado por Muga y Sotomayor (Lemiatre, 2017), se sugiere que, dependiendo del adecuado nivel de implementación de estos mecanismos e instrumentos, y del grado de eficacia con que lo comunique a los diferentes grupos de interés, las universidades pueden ser más activas tanto en dar cuenta del nivel de calidad alcanzado, como en la construcción de su reputación institucional. El análisis realizado permite suponer que existe una articulación entre aseguramiento de la calidad y reputación institucional. En este sentido el Modelo Clark-Engwall-Soto permite comprender mejor las dimensiones de la reputación institucional, aspecto poco tratado en la literatura del área, y como las instituciones pueden trabajar en la consolidación de su reputación.

Al identificar los instrumentos de política pública, así como los productos disponibles que articulan las relaciones entre los diferentes componentes del modelo que denominamos Clark-Engwall-Soto, permite por una parte entender cómo se fortalece o debilita la posición de las IES a partir de sus interacciones con el Estado-Regulador, los mercados y la sociedad civil. Contrario a lo planteado por Clark (1983) y revisado por Brunner (2019), el peso de las oligarquías académicas ha disminuido en la actualidad en la mayoría de los países de América Latina, especialmente por la reducción del tamaño de la matrícula de las universidades públicas frente al de las privadas, las que introducen modelos de gobernanza diferentes, que conceden menos peso a estas oligarquías. Solo en los movimientos estudiantiles en Colombia es posible deducir la actuación de algunos profesores a través de las protestas estudiantiles, especialmente en los movimientos del 2018, y mucho menos en los del 2021. Lo que se expresó en el 2018 en los petitorios estudiantiles y en la participación de sindicatos de profesores universitarios de universidades públicas emblemáticas en los acuerdos y las comisiones de seguimiento de los mismos.

Coincidentemente con Lemiatre (2017), la calidad, en cuanto aspiración compartida por la sociedad, es una temática de constante discusión, objeto de estudio y políticas en constante reformulación. Existe un cierto consenso en que el sistema de aseguramiento de la calidad debe mantener atención tanto por la consistencia interna de la oferta académica en la medida que ésta se alinea a los propósitos institucionales y a la promesa que se hace a los alumnos; como por la consistencia externa, consistente en la pertinencia de los propósitos en respuesta a las demandas de la sociedad. 
Desde el punto de vista de las IES el aseguramiento de la calidad ha estado cargado de desafíos y dificultades (Lemaitre, 2017). En muchas instituciones emergen tensiones entre la autoevaluación y la cultura institucional: la autoevaluación es percibida a veces como más propia de la administración que del mundo académico, o como una tarea inherente a la función docente a la cual la investigación no está llamada. Esto último porque los mecanismos de aseguramiento de la calidad científica dependen de la revisión de pares en las comunidades disciplinares, y no pasa por el juicio de los no científicos. Desde el punto de vista del EstadoRegulador también se aprecian inconsistencias y fragmentaciones. Para los actores de los mercados, las decisiones que deben tomar respecto de las instituciones no sólo están dificultadas por la falta de información, sino también por un problema de credibilidad, de confianza en su conducta futura. En este sentido las propias IES pueden ser activas de modo individual y colectivo.

En América Latina lo observado por Engwall (2020) en Europa, se está cumpliendo. La Sociedad Civil a través de agentes privados que escudriñan, examinan, inquieren, averiguan, y comunican sus análisis están modificando la percepción de reputación de las instituciones y por tanto inciden sobre la gobernanza universitaria. Ellos actúan tensionando la forma que las IES muestran consistencia con los propósitos y promesas contraídas con la comunidad universitaria, como las realizadas en forma explícita o tacitas con la sociedad, los mercados y el entorno. El modelo propuesto permite entender mejor como las IES pueden construir, cuidar y gestionar la reputación institucional. Toda la comunidad institucional genera o destruye la reputación institucional, sin embargo, los estudiantes son un agente clave en su construcción.

\section{CONCLUSIONES}

De acuerdo al trabajo presentado y a los resultados obtenidos, se pueden plantear las siguientes conclusiones principales: En países donde una baja proporción de las IES públicas estén acreditadas, especialmente si es en menor medida al nivel alcanzado por las instituciones privadas, pone en duda la convicción del propio Estado-Regulador en las políticas y los instrumentos empleados para asegurar la calidad de las IES, y muestra una falta de articulación entre fines y medios; Parece ser recomendable que el Estado-Regulador analice la eficacia y el costo de las políticas, medidas, normas, y procedimientos con que grava las IES. A lo menos desde una perspectiva teórica, los Estados muestran oportunidades de mejora sus políticas tanto en la perspectiva de asegurar la calidad de los sistemas de educación superior, como en buscar el óptimo uso de los recursos públicos y privados. La calidad es necesaria y alcanzarla tiene un costo significativo; Los mercados en que participan las IES requieren de información oportuna y de calidad, generando una mayor simetría entre los diferentes actores que deben tomar decisiones. Si bien la legislación ayuda a que ella fluya mejor, y que diferentes actores asumen la tarea y el costo de organizarla y desplegarla, también parece ser deseable que los actores aprendan a leer la información disponible de forma adecuada; La Sociedad Civil ha adquirido un peso significativo, que influencia las políticas públicas, el funcionamiento de diversos mercados y la gobernanza universitaria. Las IES cada vez son más sensibles a ellas. Establecen mecanismos permanentes de escucha, implementan planes de mejora, y comunican adecuadamente sus logros; y La reputación institucional y el aseguramiento de la calidad son dos caras de la misma moneda. Sin embargo, pareciera ser que la mayoría de las IES entienden la reputación institucional como el resultado de una tarea bien hecha, más que una dimensión que pudiera gestionarse. El modelo propuesto pone en evidencia que la reputación es gestionable en un marco de cultura universitaria, buenas prácticas y ética.

\section{REFERENCIAS}

Bosi, L., Giugni, M., y Uba, K., The Consequences of social movements, https://doi.org/10.1017/CBO9781316337790, Cambridge, UK., Cambridge University Press (2016)

Brooks, R., Gupta, A., y otros tres autores, Students as political actors? Similarities and differences across six european nations, https://doi.org/10.1002/berj.3628, British Educational Research Journal, 46 (6), 1193-1209 (2020)

Bustos-González, A., The transit of a teaching university to a research university. A problem of academic information, taxonomies or university rankings?, https://doi.org/10.3145/epi.2019.jul.22, Profesional de la Información, 28 (4), art. no. e280422 (2019)

Brunner, J.J., Vargas, J.R., y otros dos autores, Teoría del capitalismo académico en los estudios de educación superior, https://doi.org/10.24320/REDIE.2019.21.E33.3181, Revista Electrónica de Investigacion Educativa, 21 (1), art. no. 3181, pp. 1-13 (2019)

Christensen, T., Gavrila, S.G., y otros dos autores, Reputation management by Chinese universities: primary profile and comparative features, http://doi.org/10.1111/padm.12679, Public Administration, 98 (4), 1027-1043 (2020)

Clark, B., The Higher Education system. Academic organization in cross national perspective, 1-315, University of California Press, Berkeley, USA (1983)

CNA-Chile, Cuenta pública 2019, https://www.cnachile.cl/ (2019)

CNA-Chile, Barómetro de Aseguramiento de la Calidad en Educación Superior, http://www.barometro.cnachile.cl/ (2020)

CNA-Colombia, Evaluación Externa Internacional del CNA, https://www.cna.gov.co/ (2020) 
Dandala, S., Public accountability and workforce diversity in Canadian public education sector, https://doi.org/10.1080/00131911.2018.1538936, Educational Review, 72 (5), pp. 650-662 (2020)

Donaldson, L., y Davis, J. H., Stewardship theory or Agency Theory: CEO Governance and Shareholder, http://doi.org/10.1177/031289629101600103, Australian Journal of Management, 16 (1), 49-64 (1991)

Dougherty, K.J., Natow, R.S., Performance-based funding for higher education: how well does neoliberal theory capture neoliberal practice? http://doi.org/10.1007/s10734-019-00491-4, Higher Education, 80 (3), pp. 457-478 (2020)

Engwall, L., (edit.), University mission. past, present, future, http://doi.org/10.1007/978-3-030-41834-2, 1-244, Cham, Switzerland, Springer Nature AG. (2020)

Fan, M., The value pursuit and meaning reconstruction of public pedagogy integrating Clarke triangle coordination model, https://doi.org/10.26802/jaots.2018.06769, Journal of Advanced Oxidation Technologies, 21 (2), art. no. 201806769 (2018)

Ganga-Contreras, F., Hidalgo, M.E., y otros dos autores, Teoría de agencia (TA): supuestos teóricos aplicables a la gestión universitaria, https://doi.org/10.15446/innovar.v25n57.50324, Innovar, 25 (57), pp. 11-25 (2015)

Geschwind, L., y Broström, A., Managing the teaching-research nexus: ideals and practice in research-oriented universities, https://doi.org/10.1080/07294360.2014.934332, Higher education research and development, 34(1), 60-73 (2015)

Guerrero-Solé, F., Suárez-Gonzalo, S., y otros dos autores, Social media, context collapse and the future of data-driven populism, https://doi.org/10.3145/epi.2020.sep.06, Profesional de la Información, 29(5) (2020)

Lemaitre, M. J., editora, Educación Superior y Sociedad (ESS), Colección 25ํAiversario UNESCO-IESALC, 1-40, (2017)

Maduro, S., Fernandes, P.O., y Alves, A., Management design as a strategic level to add value to corporate reputation competitiveness in higher education institutions, https://doi.org/10.1108/CR-04-2017-0029, Competitiveness Review, 28 (1), 75-97 (2018)

Muga, A., Sistemas de información para la gestión universitaria. La visión de la agencia central que asigna recursos en una organización descentralizada. 256-276. CINDA, https://cinda.cl/ (1997)

Muga, A., La información: ¿referencia de catálogo o material de caja de herramientas? https://www.cned.cl/, Revista Seminarios / CSE-CNAP, (5), 62-68. (2001)

Qureshi, M.S., Daud, A., y otros dos autores, OpenRank - a novel approach to rank universities using objective and publicly verifiable data sources, https://doi.org/10.1108/LHT-07-2019-0131, Library Hi Tech (2021)

Rider, S., Peters M.A., y otros dos autores, World Class Universities. Evaluating education: normative systems and institutional practices, https://doi.org/10.1007/978-981-15-7598-3, 1-287, Springer, Singapore (2020)

Schwartzman, S. (edit.), Higher education in latin america and the challenges of the 21st century, 1-162, Springer Nature AG, Cham, Switzerland, https://doi.org/10.1007/978-3-030-44263-7, (2020)

Shu-Ling-Huang, S., y Shen, S.P., Relationships among academic ranks, numbers of funded projects, and research productivity of university faculty: the case of a private medical university, https://doi.org/10.6209/JORIES.2017.62(4).04, Journal of research in education sciences, v. 62, n. 4, 89-115 (2017)

Torabian, J., Revisiting global university rankings and their indicators in the age of sustainable development, https://doi.org/10.1089/sus.2018.0037, Sustainability (United States), 12 (3), 167-172 (2019)

Tuesta, E.F., Bolaños-Pizarro, M., y otros tres autores, Complex networks for benchmarking in global universities rankings, https://doi.org/10.1007/s11192-020-03637-9, Scientometrics, 125 (1), 405-425 (2020)

Vetrova, E.A., Kabanova, E.E.,y otros dos autores, Management of educational services promotion in the field of higher education (the example of "Russian State Social University"), https://doi.org/10.13187/ejced.2019.2.370, European Journal of Contemporary Education, 8 (2), 370-377 (2019)

Vincent, J., Lee, J.W., y otros dos autores, Where legends are made: a case study of an advertising and branding campaign at the University of Alabama, https://doi.org/10.1123/ijsc.2019-0098, International Journal of Sport Communication, 13 (1), 97-128 (2020)

Zhou, Z., The development strategy research of higher education management from the perspective of "Internet +", https://doi.org/10.1007/978-981-33-4572-0_65, Advances in Intelligent Systems and Computing, 1303, 444-450 (2021) 